\title{
Outcomes of cardiopulmonary resuscitation in the emergency department
}

\author{
Seyed Hossein Ojaghi Haghighi' ${ }^{1}$, Samad Shams Vahdati ${ }^{2}$, Tarannom Mahmoudie', Pegah Sepehri Majd ${ }^{1}$, \\ Mohammad Mirza-Aghazadeh-Attari ${ }^{3}$
}

'Emergency Medicine Research Team, Tabriz University of Medical Sciences, Tabriz, Iran

${ }^{2}$ Road Traffic Injury Research Center, Tabriz University of Medical Sciences, Tabriz, Iran

${ }^{3}$ Medical Philosophy and History Research Center, Tabriz University of Medical Sciences, Tabriz, Iran

Received: 13 February 2017
Accepted: 30 March 2017
Published online: 2 May 2017
*Corresponding author: Samad
Shams Vahdati; Ghoghasht Street,
Emergency Department, Imam Reza
Hospital, Tabriz, Iran.
Tel: +98-4133352078
Email: pegah.sm87@gmail.com
Competing interests: None.
Funding information: None.
Citation: Ojaghi Haghighi SH,
Shams Vahdati S, Mahmoudie
T, Sepehri Majd P, Mirza-
Aghazadeh-Attari M. Outcomes of
cardiopulmonary resuscitation in
the emergency department. Journal
of Emergency Practice and Trauma
2017; 3(2): $49-52$. doi: $10.15171 /$
jept.2017.14.

\begin{abstract}
Objective: Cardiopulmonary resuscitation (CPR) is a lifesaving technique useful in the prevention of death or delaying it in a person with cardiac arrest. In this regard, demographic information about patients who need CPR is vital.

Methods: In this cross-sectional study patients with cardiopulmonary arrest or arrhythmias admitted to Imam Reza and Sina educational hospitals of Tabriz University of Medical Sciences from 22 December 2013 to 21 December 2014 entered the study. Demographic information such as age, sex, cardiopulmonary resuscitation time, the place of cardiopulmonary arrest (outside or inside the hospital), the duration of resuscitation process, success or failure of the resuscitation process and the mechanism of cardiopulmonary arrest were obtained.

Results: From a total of 354 cases of cardiopulmonary resuscitation, 281 cases (79\%) were unsuccessful and 73 cases $(21 \%)$ were successful. The average age of patients was $59 \pm$ 22 years. The average time of the resuscitation process was $31 \pm 12$ minutes. There was a significant difference between the mean of age and resuscitation time in patients who had experienced successful or unsuccessful resuscitation $(P=0.0001)$. There was a significant relationship between sex and the success rate of resuscitation $(P=0.0001)$. In addition, a significant relationship between the success of the resuscitation operation and the ward of resuscitation was observed $(P=0.0001)$.

Conclusion: The most common mechanism leading to cardiopulmonary arrest among patients was asystole. In this regard, no significant difference was observed between successful and unsuccessful resuscitation processes. It was also observed that the success of resuscitation from 8 AM to 4 PM was more than any other time period.

Keywords: Cardiopulmonary resuscitation (CPR), Cardiac arrest, Emergency department
\end{abstract}

\section{Introduction}

Cardiopulmonary resuscitation (CPR) is an emergency procedure to restore vital functions of 2 important organs, the heart and lungs. CPR is an effort to restore spontaneous blood circulation and breathing in a person with cardiac arrest. Noteworthy, because without these actions due to lack of oxygen, permanent brain death within less than 4-6 minutes (the golden time) develops. The term CPR on a proposal by Peter Safar (a key frontier and the father of $\mathrm{CPR}$ ), due to the importance of restoring and maintaining the functioning of the brain, was renamed to the cerebral cardiopulmonary resuscitation (CPCR) (1,2). In the past, CPR was performed using the air force through the mouth, chest pressure and sometimes physically painful stimuli. But in the course of time with the progression of science, there has been developments regarding this procedure. Today, CPR is preformed using medication and advanced tools, and the patient is revived during 2 stages of the initial basic life support measures (basic life support) and advanced maintenance measures (advanced cardiac life support) (3). According to the American Heart Association, fast initiation of CPR within 3 to 5 minutes after cardiac arrest and also the initiation of advanced life support not only increases the survival rate but also decreases long-term consequences in patients more than $50 \%(4,5)$. Unfortunately, the success rate of CPR has remained constant over 30 years (6). Another grim fact is that the standard procedure of CPR is not implemented in many cases (because of technical deficits or uneducated work force) (7), and this leads to lower survival rates among patients. Studies have shown the effects of the trained people on the resuscitation process. They indicated that an expert can have a significant effect on the outcome of resuscitation $(8,9)$. According to the Iranian Ministry of Health and Medical Education, cardiovascular disease is known as the first cause of mortality in the country with more than 300000 death tolls per year. Research shows that $20 \%$ to $30 \%$ of these patients are resuscitated. But no 
data is available about the success rates of the procedure and the demographic information of the patients makes further evidence-based decision making impossible (10). Therefore, we aimed to cover this informational gap in the north-west region of Iran by extracting demographic information of the patients hospitalized in educationalmedical centers of Tabriz University of Medical Sciences.

\section{Methods}

In this cross-sectional study, the medical records of all patients who received cardiopulmonary resuscitation in Imam Reza and Sina educational hospitals of Tabriz University of Medical Sciences were reviewed. Before study initiation, permission from the Deputy of Research and Technology and confirmation of Regional ethics committee was obtained.

Inclusion criteria included unconscious patients, patients with abnormal breathing and patients with a positive diagnostic evaluation of cardiopulmonary arrest by the emergency medical team or the referring physician. Exclusion criteria consisted of children under 8, patients without a medical record, intoxication, trauma, CPR cases done not by a specialist or an emergency medical staff, and patients with obvious signs of death before the initiation of CPR.

CPR was performed in accordance to the most recent guidelines. In each CPR performed, there was a rapid response team present $(11,12)$. This team consisted of a physician, senior resident or intensivist or hospitalist, physician's assistant, critical care specialist, clinical nurse specialist and respiratory therapist.

Study variables included age, sex, CPR referral time, month, time of cardiopulmonary resuscitation, place of cardiopulmonary arrest (inside or outside the hospital), duration of resuscitation process, the success or failure of resuscitation, ward of resuscitation and the mechanism of cardiopulmonary arrest. The questionnaire which was used to gather data was completed by researchers at designated hospitals of Tabriz University of Medical Sciences.

Statistical analysis was performed by Statistical Package for the Social Sciences (SPSS) software package version 16.0 (SPSS Inc., Chicago, USA). Quantitative data were presented as mean \pm standard deviation (SD), while qualitative data were demonstrated as frequency and percent (\%). For statistical analysis, after determining the distribution of continuous variables by KolmogorovSmirnov test, independent sample $t$ test was applied to compare 2 groups of data. Also, collected data were analyzed using descriptive statistical methods. The mean difference test for independent groups, chi-square test or Fisher exact test were used accordingly. $P$ value less than 0.05 was considered as statistically significant.

\section{Results}

This study was conducted at 2 educational hospitals of Tabriz University of Medical Sciences (Sina and Imam Reza hospitals). Totally, 354 cases had CPR. Of these cases, 325 and 29 cases received CPR in Imam Reza hospital and Sina hospital respectively. The mean age of patients was $59 \pm 22$ years. The maximum age recorded was related to a 102-year-old man and the minimum age recorded was related to a 3-year-old girl. A total of 137 patients were female (39\%) and 214 were male (60\%). The average time of the resuscitation process was $31 \pm$ 12 minutes. Minimum resuscitation time was 5 minutes and maximum time was 120 minutes. From the total of 354 cases resuscitated in Imam Reza hospital, 255 cases were unsuccessful and 70 cases were successful. In Sina hospital, 23 cases experienced unsuccessful resuscitation and 3 cases had successful resuscitation. From the total of 354 cases (both Imam Reza and Sina hospitals), 281 cases (79\%) had unsuccessful resuscitation and 73 cases (21\%) had successful resuscitation. From the successful CPRs performed, except 12 cases, all were performed during the morning shift between $8 \mathrm{AM}$ and $4 \mathrm{PM}$ and the rest were done in the night shift. There was a significant difference in this regard $(P<0.002)$. Findings showed that 202 cases $(59 \%)$ of cardiopulmonary arrest occurred in the hospital and 139 cases (41\%) occurred outside the hospital. Among the patients who experienced cardiopulmonary arrest outside the hospital, resuscitation in 111 cases $(80 \%)$ was unsuccessful and 28 cases (20\%) had successful resuscitation. Among the patients who experienced cardiopulmonary arrest inside the hospital, 157 cases (78\%) were unsuccessful and 45 cases (22\%) were successful. There was a significant difference between the successful and unsuccessful resuscitation in patients experiencing a cardiopulmonary arrest inside or outside the hospital $(P=0.0001)$. Among the cases with unsuccessful resuscitation, 168 cases were male $(60 \%)$ and 110 cases were female $(40 \%)$. The average age of these patients was $60 \pm 21$ years. The average duration of the resuscitation process for them was $33 \pm 10$ minutes. Among all the patients with unsuccessful CPR, in 241 cases $(76.85 \%)$, there was an asystolic rhythm. 23 cases (18.8\%) had pulseless cardiac activity and 14 cases (9.84\%) had ventricular tachycardia. Concerning the successful resuscitation, 46 cases (63\%) were male and 27 cases (36\%) were female. The average age of these patients was $55 \pm 30$ and the average duration of resuscitation for them was 21 \pm 16 minutes. Among these patients, 51 cases had asystole rhythm $(86.69 \%), 15$ cases had ventricular tachycardia (54.20\%) and 7 cases had ventricular fibrillation (58.9\%). There was a significant difference between the mean age and the time spent on resuscitation in patients who had successful or unsuccessful resuscitation $(P=0.0001)$. There was also a significant relationship between sex and the success rate of resuscitation $(P=0.0001)$. In addition, there was a significant relationship between the success of resuscitation and the hospital where the procedure was done $(P=0.0001)$.

\section{Discussion}

Our study showed that the success rate of cardiopulmonary resuscitation was $20 \%$ to $22 \%$. We found that the success 
rate of CPR in intensive care unit (ICU) as 3.3\% and the other wards was $14 \%$. According to the data obtained in our study can be seen, despite workload and frequency of cardiorespiratory arrest occurred at $8 \mathrm{AM}-4 \mathrm{PM}$ of the day our success rate is considerably higher than the other hours of the day. This can be related to the number of working personnel as well as the energy and motivation of medical staff. There is a need for more research and documents in this regard. In our research success rate in the process of CPR in patients with cardiac arrest had been in the emergency center significantly more than was taken outside the medical center. Also our study observed a significant difference in the time of resuscitation process in some cases, unsuccessful resuscitation was significantly less than successful resuscitation, this may be due to the patient's clinical conditions, medical staff mood and other factors. In our study was observed most patients who have a cardiopulmonary arrest had asystole rhythm; and a smaller percentage of patients had other arrhythmias. Due to the low number of patients in our study how has experienced ventricular fibrillation or ventricular tachycardia thus there was not possible to reviewed among different rhythms. Kazaure et al studied the epidemiology and outcome of in-hospital CPR in the United States between 2000 and 2009 and found that CPR was conducted more in clinical wards. CPR rate increased $33.7 \%$ during the study (from one in every 453 patients to one in every 339 patients). They also found that the median age of patients decreased during 10 years (in $2000,33.4 \%$ of patients were over 65 years whereas in the year 2009 it was $40.0 \%$ ). During these years, the discharge rate to hospice increased significantly from $0.4 \%$ to $7.1 \%$. The success rates of CPR also increased from $19.9 \%$ to $25.8 \%$ in men and from $21.3 \%$ to $27.2 \%$ in women (13). It can be seen that the survival rate of CPR was higher in this study compared to our study (21\%), possibly due to better staff management and technical superiority in the United States. In this study, 55\% of patients were male. Stapleton et al conducted a study to compare the result of CPR in patients with or without chronic diseases and found that patients suffering from chronic illnesses had a smaller chance of surviving cardiopulmonary arrest $(P$ $<0.001$ ) (14). This was also the case in our study, but exact information is needed to make comparisons. In addition, there is ambiguity about the definition of chronic diseases as some patients may be in the early phases of their disease. Youness et al conducted a review on patients receiving CPR and concluded that the mean time of CPR was 75 minutes with a range of 20 to 330 minutes. Mean CPR time in the present study was $31 \pm 10$ minutes. The initial cardiac arrest rhythm was ventricular tachycardia or fibrillation in 41 cases (50\%), and pulseless electrical activity or asystole in 37 cases (45\%). In our study the most common rhythm was the asystole rhythm which is inconsistent with their result (15). The survival rate of CPR was $83 \%$ compared to $21 \%$ in our study. This difference can be due to the higher length of resuscitation process and the age difference between the 2 studies $(43 \pm 21$ years compared to $59 \pm$
22 years). Pearson et al compared the efficiency of teambased CPR with out of hospital CPR and found that the outcome of team-based CPR was much more favorable (836 cases of good neurological outcome (8.3\%, 95\% CI: $7.7 \%-8.8 \%)$ in team-based in-hospital CPR vs. 193 (4.8\%, 95\% CI: $4.2 \%-5.5 \%$ ) of standard out of hospital CPR) (16). This was in line with our study, which showed higher rates of success in team-based in-hospital CPR initiation. Miranzadeh et al studied the survival rate of CPR among 238 patients. They reported the survival rate at $4.8 \%$. The mean duration of CPR was $35.11 \pm 11.42$ minutes. The survival rate was higher in morning shifts, with no patient surviving CPR in the afternoon shift. This can be owing to higher concentration and stamina of the work force in the morning (17). Likewise, there was a significant difference between the shifts in our study $(P<0.002)$. Miranzadeh et al concluded that the predicting factors of CPR success were the duration and the urgent initiation of CPR. One important difference seen in the studies and previously noted is the rather high difference between the success rates of different studies (18) which could not solely due to staff differences. In our study we only considered the initial success of CPR procedure. We believe that this may have a great effect on the final result. For example, Goodarzi et al (19) studied the survival rate of CPR for 6 months and found that the survival rate decreased from $15.3 \%$ to $8.78 \%$.

This study has some limitations. Our data was gathered by referring to patients' medical records. Also, in some cases the final result of resuscitation was not available to researchers. Thus it is obvious prospective studies reinforced with functional universal health accounts recording the long term outcome of CPR performed on patients would be the most beneficial.

\section{Conclusion}

The findings of the study showed more significantly difference in the success rate of resuscitation in patients with cardiopulmonary arrest in the emergency department than patients who have cardiopulmonary arrest outside the emergency department. The most common mechanism leading to cardiopulmonary arrest among patients was asystole. In this regard, we did not observe a significant difference between successful and unsuccessful resuscitation processes. It was also observed that the success of resuscitation from 8 AM to 4 PM was more than any other time of the day.

\section{Ethical issues}

All data were collected from patients' medical records. No type of diagnostic or therapeutic intervention was performed on patients by the researchers. Patients' information was kept confidential and the anonymity of patients was guaranteed during the study. The study protocol was in accordance with the ethical guidelines of Tabriz University of Medical Sciences and the Helsinki Declaration. 


\section{Authors' contributions}

SHOH: Study design and suppervision; SSV: analysis and critic evaluation; TM: Data ghathering; PSM: article review and data ghathering; MMA: writting.

\section{References}

1. Shiri H, Golshani Golbaghi G, Nikravan Mofrad M. Comprehensive CPR in Adult. Tehran: Noore-Danesh Publication; 2001. p. 1-5. [In Persian].

2. Olotu A, Ndiritu M, Ismael M, Mohammed S, Mithwani $\mathrm{S}$, Maitland $\mathrm{K}$, et al. Characteristics and outcome of cardiopulmonary resuscitation in hospitalised African children. Resuscitation 2009; 80(1): 69-72. doi: 10.1016/j. resuscitation.2008.09.019.

3. Lee K. Cardiopulmonary resuscitation: new concept. Tuberc Respir Dis (Seoul) 2012; 72(5): 401-8. doi: 10.4046/ $\operatorname{trd}$.2012.72.5.401.

4. Rakić D, Rumboldt Z, Carević V, Bagatin J, Polić S, Pivac N, et al. In-hospital cardiac arrest and resuscitation outcomes: rationale for sudden cardiac death approach. Croat Med J 2005; 46(6): 907-12. doi: 10.1161/hc4701.099784.

5. Kim C, Fahrenbruch CE, Cobb LA, Eisenberg MS. Outof-hospital cardiac arrest in men and women. Circulation 2001; 104(22): 2699-703.

6. Bellomo R, Goldsmith D, Uchino S, Buckmaster J, Hart GK, Opdam $\mathrm{H}$, et al. A prospective before-and-after trial of a medical emergency team. Med J Aust 2003; 179(6): 283-8.

7. Shams Vahdati S, Ojaghi Haghighi S, Paknejad P, Fahimi R, Tajoddini S. One year evaluation of trauma patients' death. Journal of Emergency Practice and Trauma 2016; 2(2): 312. doi: $10.15171 /$ jept.2016.07.

8. Tyrer F, Williams M, Feathers L, Faull C, Baker I. Factors that influence decisions about cardiopulmonary resuscitation: the views of doctors and medical students. Postgrad Med J 2009; 85(1009): 564-8. doi: 10.1136/pgmj.2009.079491.

9. Salari A, Mohammad Nejad E, Vanaki Z, Ahmadi F. Effect of in-hospital cardiopulmonary cerebral resuscitation management on resuscitation outcomes. J Crit Care Nurs 2011; 4(1): 13-22.

10. Christenson J, Nafziger S, Compton S, Vijayaraghavan K, Slater B, Ledingham R, et al. The effect of time on CPR and automated external defibrillator skills in the Public Access Defibrillation Trial. Resuscitation 2007; 74(1): 52-62. doi: 10.1016/j.resuscitation.2006.11.005.

11. Ghaffarzadeh A, Shams Vahdati S, Salmasi S. Assessment of emergency medicine residents' cardiopulmonary resuscitation team in imam reza hospital. J Cardiovasc Thorac Res 2012; 4(3): 85-6. doi: 10.5681/jcvtr.2012.021.

12. Benin AL, Borgstrom CP, Jenq GY, Roumanis SA, Horwitz LI. Defining impact of a rapid response team: qualitative study with nurses, physicians and hospital administrators. BMJ Qual Saf 2012; 21(5): 391-8. doi: 10.1136/ bmjqs-2011-000390.

13. Kazaure HS, Roman SA, Sosa JA. Epidemiology and outcomes of in-hospital cardiopulmonary resuscitation in the United States, 2000-2009. Resuscitation 2013; 84(9): 1255-60. doi: 10.1016/j.resuscitation.2013.02.021.

14. Stapleton RD, Ehlenbach WJ, Deyo RA, Curtis JR. Longterm outcomes after in-hospital CPR in older adults with chronic illness. Chest 2014; 146(5): 1214-25. doi: 10.1378/ chest.13-2110.

15. Youness $\mathrm{H}$, Al Halabi $\mathrm{T}$, Hussein $\mathrm{H}$, Awab A, Jones K, Keddissi J. Review and outcome of prolonged cardiopulmonary resuscitation. Crit Care Res Pract 2016; 2016: 7384649. doi: 10.1155/2016/7384649.

16. Pearson DA, Nelson RD, Monk L, Tyson C, Jollis JG, Granger CB, et al. Comparison of team-focused CPR vs standard CPR in resuscitation from out-of-hospital cardiac arrest: results from a statewide quality improvement initiative. Resuscitation 2016; 105: 165-72. doi: 10.1016/j. resuscitation.2016.04.008.

17. Miranzadeh S, Adib-Hajbaghery M, Hosseinpour N. A prospective study of survival after in-hospital cardiopulmonary resuscitation and its related factors. Trauma Mon 2016; 21(1): e31796. doi: 10.5812/ traumamon.31796.

18. Tavakoli N, Bidari A, Shams Vahdati S. Serum cortisol levels as a predictor of neurologic survival in successfully resuscitated victims of cardiopulmonary arrest. J Cardiovasc Thorac Res 2012; 4(4): 107-11. doi: 10.5681/jcvtr.2012.026.

19. Goodarzi A, Jalali A, Almasi A, Naderipour A, Kalhori RP, Khodadadi A. Study of survival rate after cardiopulmonary resuscitation (CPR) in hospitals of Kermanshah in 2013. Glob J Health Sci 2014; 7(1): 52-8. doi: 10.5539/gjhs. v7n1p52. 